\title{
What's going on in there?
}

\author{
L. Pullum Australia
}

\begin{abstract}
Paste and thickened tailings both share similar properties, in that they appear to behave like homogeneous non-Newtonian fluids. Despite several publications on the issue, including various presentations at Paste conferences, the stratification of these materials, when subjected to a constant shear field, such as a laminar pipeline flow, still seems to be generally unknown. Design engineers design lines based on simple, small scale, rheological studies, using the assumption of homogeneous flow and the simplicity of a laminar flow regime. Such procedures generally under predict full scale operation, making large, or even full scale testing, appear to be essential. A review is given in this paper on research conducted by various groups, on such flows, in recent years, and describes methods that allow small scale testing to accurately predict full scale behaviour.
\end{abstract}

\section{Introduction}

This paper provides a somewhat one-eyed review of the author's understanding of paste and thickened tailings behaviour and reports on recent developments by various research teams in probing these flows.

A common problem in understanding these flows is that of dynamic similitude, and that a material with a $20 \mathrm{~Pa}$ yield stress may behave in a similar manner to one that has a $200 \mathrm{~Pa}$ yield stress, if analysed correctly. This misunderstanding is also complicated by a lack of generality that places people into various camps. Thickened tailings are different to pastes, which are different to stabilised flows. While this is obviously true in some regard, there is also a common generality that links all of these flows, namely, the transport of a particulate phase in a non-Newtonian fluid. This includes the case where, if the particles are small enough, the particulate phase combines with the fluid to form a new non-Newtonian slurry. However, it is the author's contention that, with the exception of some truly colloidal suspensions, most industrial suspensions, and certainly the suspensions which are the subject of this seminar, contain 'coarse particles'. 'Coarse', in this sense, means particles that are too massive to be intimately combined with the fluid to form a new single phase, arguably particles that are greater than $20 \mu \mathrm{m}$. The author hopes to demonstrate this by presenting results obtained from all of these "individual" flows, starting with a viscous paste.

\subsection{Case study - clay based mine backfill}

From a hydraulic conveying perspective, paste and thickened-tailings disposal appears to be a far simpler proposition than pumping conventional tailings: there is no need for the design engineer to worry about minimum conveying velocities, or concentration profiles and the flow is laminar, so that simple analytical equations can be used to calculate the flow's behaviour, once the tailings have been rheologically characterised. Consider the backfill paste shown in Figure 1.
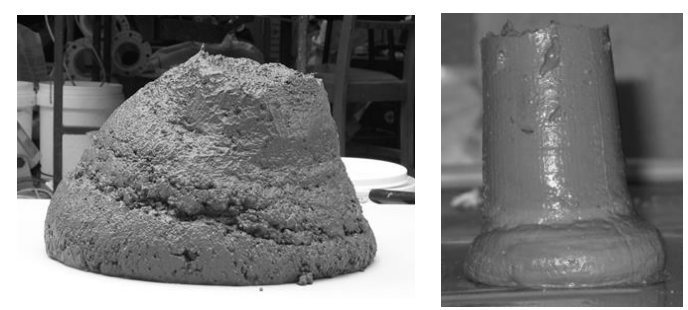

Figure 1 Slump tests for backfill paste. LHS before pumping, RHS during pumping, showing evidence of clay liberation 
This clay-based paste is typical of many pastes, when they are used. It is far more viscous than thickened tailings, having a dynamic shear stress of around 200-300 Pa; it has a broad size distribution containing a relatively small amount of particles in excess of $500 \mu \mathrm{m}$, and, like thickened tailings, it is stable, showing no signs of bleeding, settling or segregation in this test.

This material and several others reported here, was tested in a $100 \mathrm{~mm}$ pipe loop, details of which are given below.

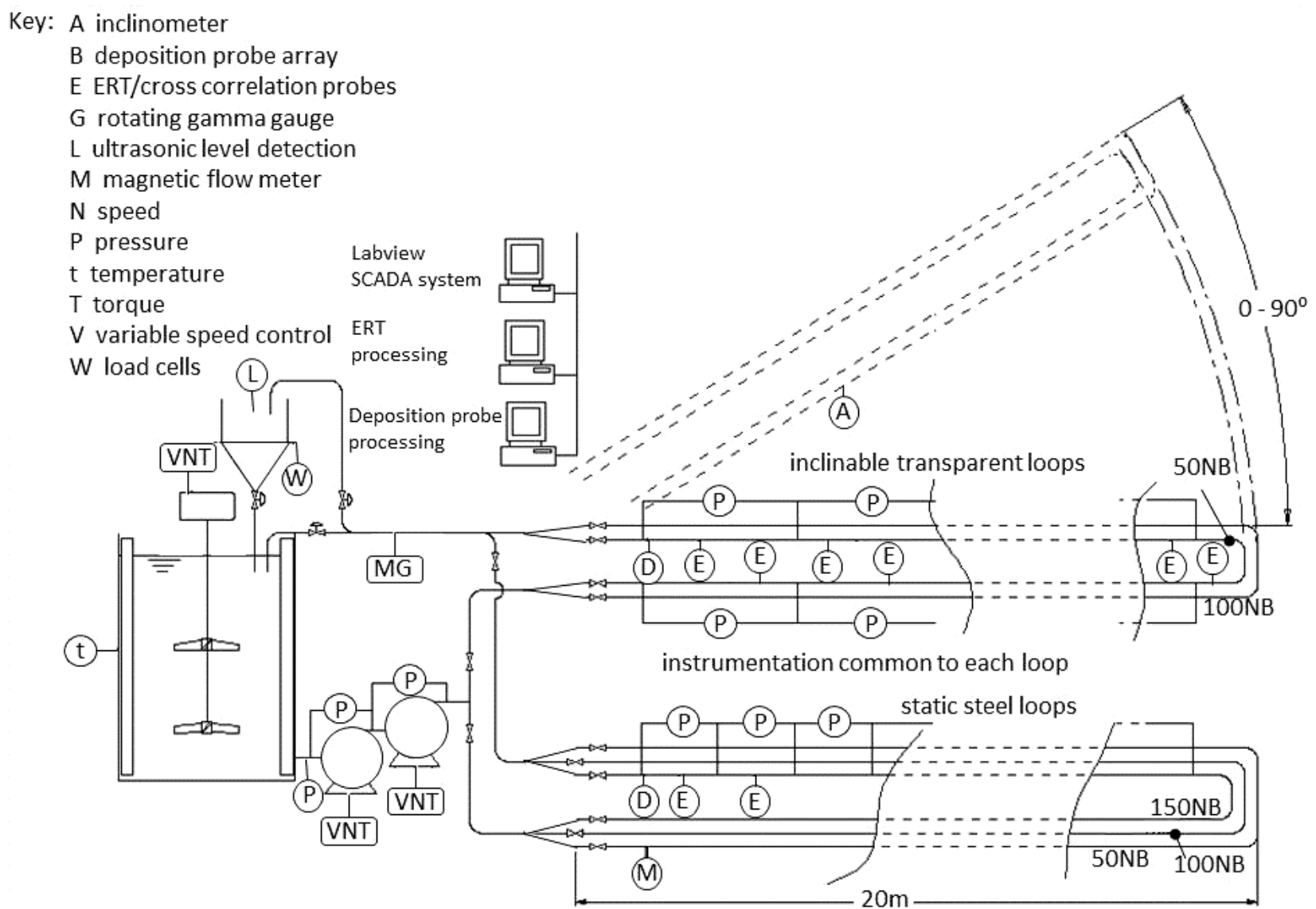

Figure 2 Schematic of CSIRO's pipe test loop facility

This loop has two centrifugal pumps in series: a Warman 4×3AH and a GIW 4×3 LCC-M, both of which are equipped with variable speed drives. The Warman pump is a specially modified froth pump, with a large inlet that allows suspensions with a high yield stress, such as this paste, to be pumped. Transport characteristics of this material for a variety of pipe inclinations are shown in Figure 2, along with the predicted behaviour based on the material's 'pseudo-rheology'. 


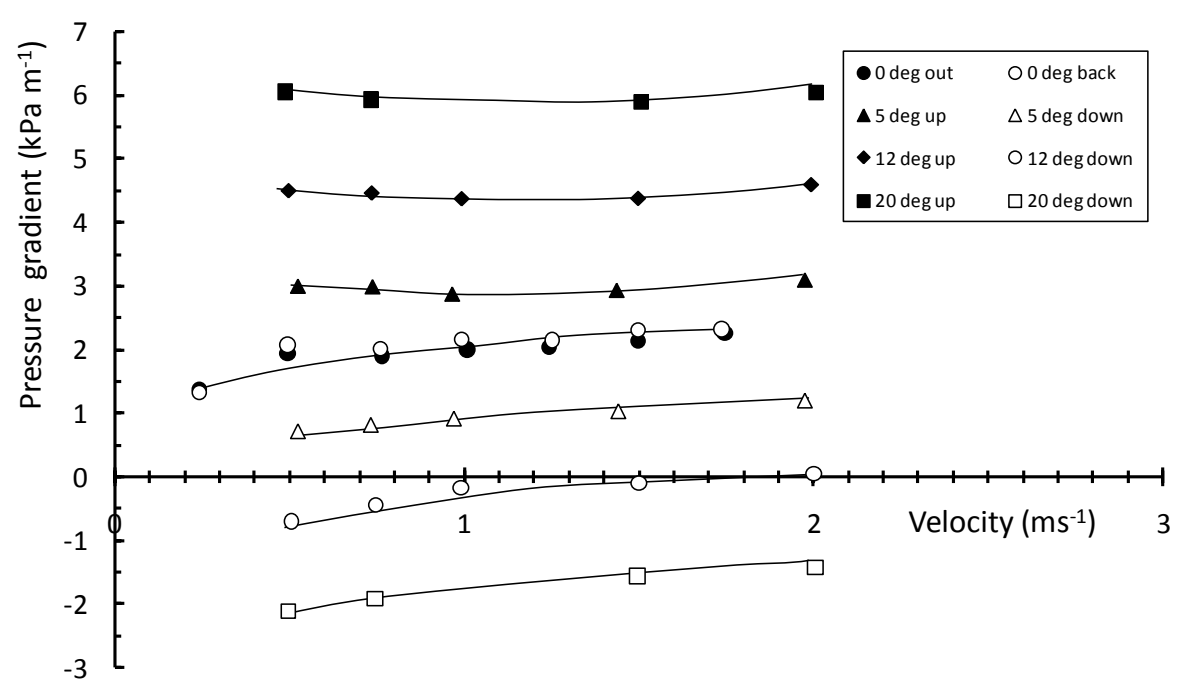

Figure 2 Transport characteristics of the backfill paste for various pipe inclinations, 100 ND pipe

The pastes' pseudo-rheology was determined from one of the horizontal pipe tests, using conventional capillary tube viscometer methods. These parameters were then used to predict the remaining data and, as shown, the predictive powers of such a model appear to be remarkably good. This method was the preferred approach in the 1980s, when Stab-Flo was a subject of considerable research activity (Brown, 1988; Duckworth et al., 1982, 1986a; Hou, 1986; Lawler et al., 1978; Lockyear et al., 1984; Pullum and Littlejohn, 1986; Thomas et al., 2004) and is still the most common method employed in designing these types of lines today. However, as we will see, since this method is mechanistically flawed, the scale up capability is very limited, and, to the author's knowledge, has only successfully been used in scaling up to pipes of order twice the test pipe's size.

The evidence that such modelling is flawed becomes apparent when the solids concentration profile is measured inside the pipe and in a region of established flow (Figure 3).

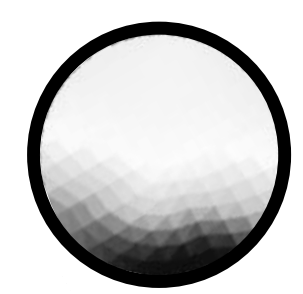

Figure 3 Solids' concentration map of the back fill paste obtained using electrical resistance tomography (ERT) at a location 200D downstream of the pump (high solids concentration denoted by dark shades)

Rather than the uniform concentration map demanded by such an analysis, a plane symmetric profile is observed - something is settling. To understand this behaviour, we must first examine the particle's behaviour in visco-plastic fluids, and then examine how this behaviour manifests itself in common flows, such as are typical of tailings lines.

\section{$2 \quad$ Sheared settling}

It is well known that, if the yield stress of the surrounding fluid is sufficiently large, solids that would normally settle are held in place by this yield stress and remain where they are. However, once the fluid is sheared, the structure of the surrounding fluid, and hence the local viscosity, is changed and the particle settles (see Figure 4). Such behaviour was first reported in the pipelining community by Thomas (1979), but has also been reported again in recent years (Cooke, 2002; Pullum and Graham, 1999) and other attempts to explain it have also been proffered (e.g. Talmon and Mastbergen, 2004; Wilson and Horsley, 2004). 


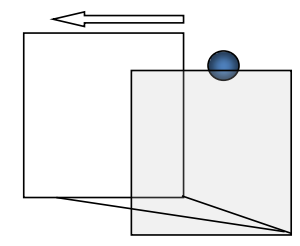

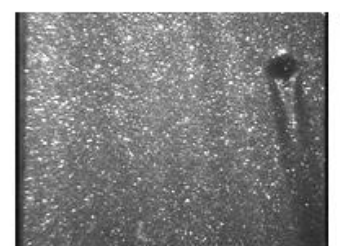

$\mathrm{t}=0 \mathrm{~s}$

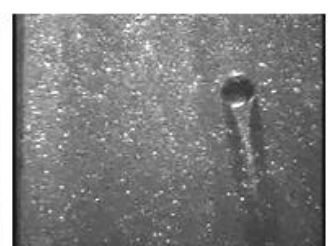

$\mathrm{t}=1.0 \mathrm{~s}$

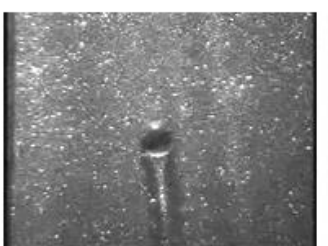

$\mathrm{t}=2.6 \mathrm{~s}$

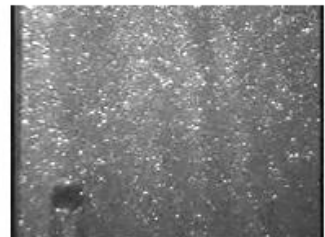

$\mathrm{t}=3.8 \mathrm{~s}$

Figure 4 Top image: a schematic of a particle settling in a planar Couette flow; bottom images: scenes taken from a video sequence demonstrating settling in a visco-plastic fluid-flow is right to left. At $t<0$ s the particle remains stationary in the unsheared fluid. At $t>0$ s the particle is subjected to shear and is convected from right to left while settling vertically

In Figure 5, a spherical particle is surrounded by a visco-plastic fluid, with a yield stress many times greater than that required to support it. Initially stationary, the particle immediately starts to settle, once the fluid is sheared. For non-Newtonian fluids, such as these, calculation of the settling velocity must consider the local shear rate around the particle, plus the shear rate imposed by the settling particle itself. When this is done, the settling velocity can be obtained as a simple function of particle Reynolds and Archimedes numbers as shown in Figure 5.

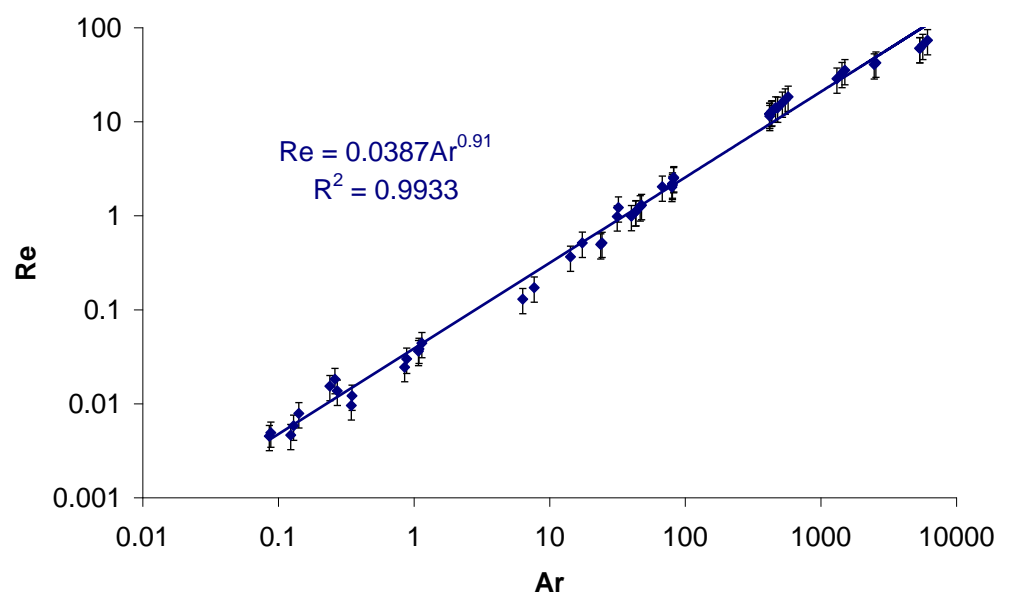

Figure 5 Variation of particle Reynolds number with Archimedes number for solids settling in visco-plastic fluids

Since visco-plastic flows are highly shear thinning, even a small amount of shear will result in a dramatic drop in local viscosity.

\section{Flows}

\subsection{Pipeline flow}

Coarse solids transported in pipelines will be subjected to the form of settling discussed above, and solids that are initially uniformly distributed, rapidly settle to form a sliding bed of material. Such behaviour has been observed in the laboratories for solids, suspended in visco-plastic fluids, with particle diameters ranging from tens of millimetres in diameter, down to solids that are less than $100 \mu \mathrm{m}$. In all cases the suspensions 
appeared to be stable and did not settle when the fluid was quiescent. In laminar flow, the only mechanisms available to re-suspend the solids are either through particle/particle impact, or through the actions of shear. For the flows considered here, the velocities, and in particular the relative velocities, are low and so the first mechanism can be ignored. The action of shear can result in viscous re-suspension and this area phenomenon has been reported by several groups (Chapman and Leighton, 1991; Charru and Mouilleron-Arnould, 2002; Krishnan and Leighton Jr., 1995; Leighton and Acrivos, 1986; Schaflinger et al., 1990, 1995; Tripathi and Acrivos, 1999; Zhang and Acrivos, 1994). In these studies the conveying fluid has always been Newtonian, but it would seem reasonable to assume that the higher viscosities, normally associated with non-Newtonian carriers, should make this a likely candidate to maintain the smaller particles in suspension. Even so, the mechanism requires a positive radial velocity gradient and so any suspension can only extend up to the dynamic centre in the pipe, i.e. mid-way between the bed surface and the top of the pipe invert, and so cannot produce homogeneity.

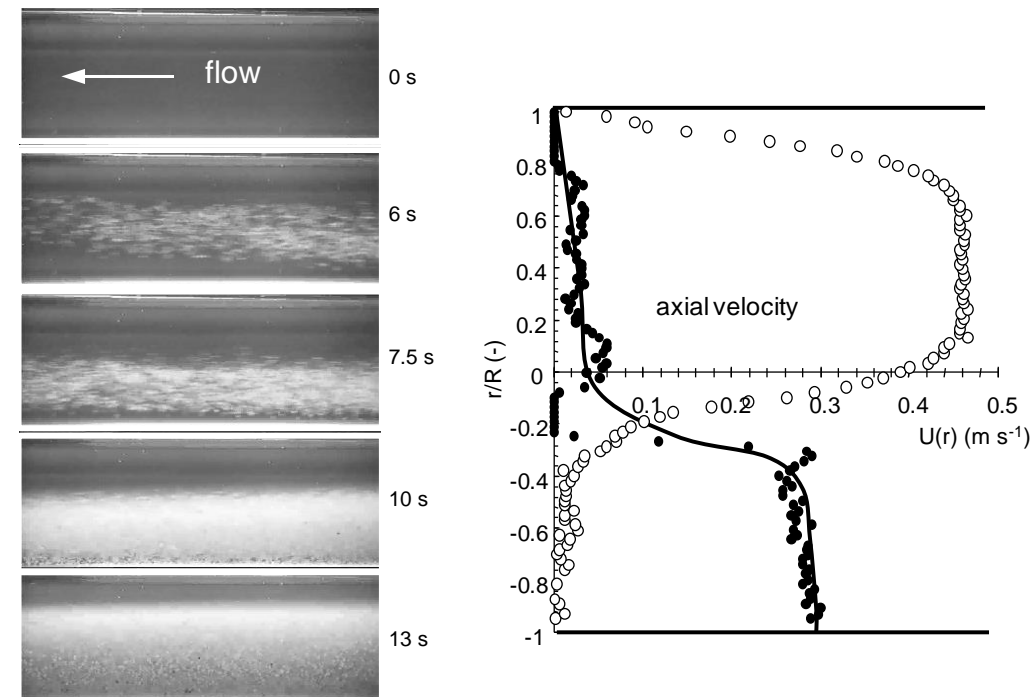

Figure 6 Images taken from a video sequence showing statically stable particles settling during
pipeline transport and fluid velocity, and solids concentration profiles obtained
downstream for this flow, using nuclear magnetic resonance (NMR) imaging

The suspension shown in Figure 6 is most closely related to thickened tailings, where the carrier fluids' rheology is comparable, but as has been shown in Section 1.1, such behaviour also occurs in pastes, where the sheared yield stresses are much, much higher.

The particles are seen to migrate across the entire pipe, and this appears to be in contradiction to the well known existence of a central unsheared plug in visco-plastic flows. If, it is argued, the solids settle due to a drop in the surrounding fluid viscosity, caused by the local shear, then in the unsheared plug, the viscosity will not change and the solids will remain resolutely suspended. However, it has been shown (Pullum et al., 2010a) that distortion of the shear field, above the proto bed of solids, will generally expose the solids originally contained in the unsheared plug to shear, thus enabling them to settle into a bed, along with those held in the sheared annulus. A smaller residual plug, of the original dispersed concentration, can sometimes be seen, depending upon ratio of the wall shear stress and carrier fluids yield stress, as shown in Figure 7. 


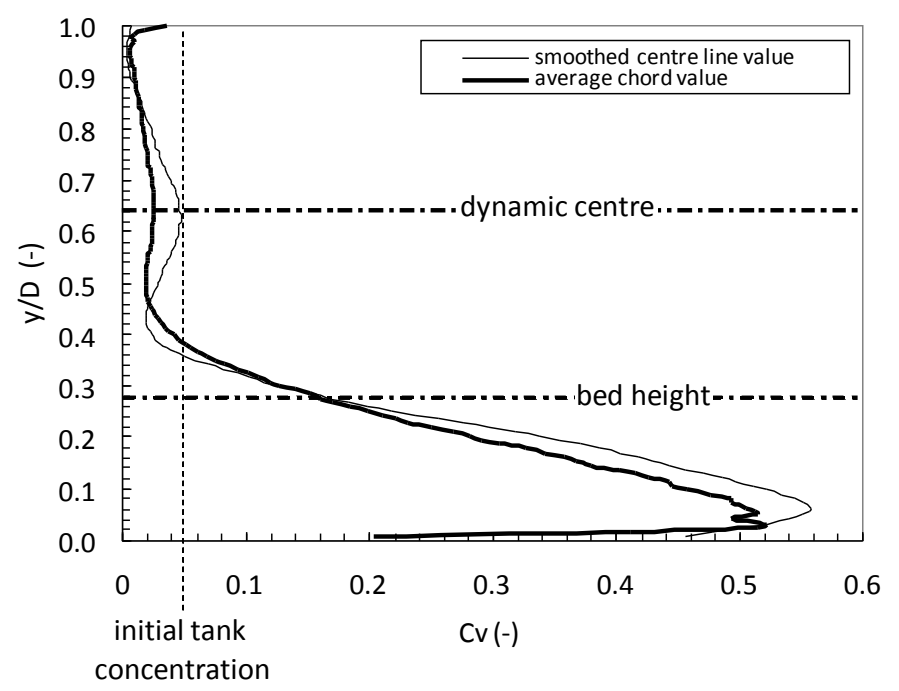

Figure 7 Settled bed concentration profiles showing evidence of a reduced residual plug. Two profiles are shown: (i) an averaged chord profile, similar to those obtained with travelling $\gamma$ gauges, and (ii), a centre line profile, that more clearly shows the residual plug positioned on the dynamic centre line (ERT imaging)

Furthermore, this bed formation is more rapid than imagined, often occurring within tens of pipe diameters, or in the case of the back fill in Section 1.1, hundreds of pipe diameters - still a relatively short finite distance.

\subsection{Pressure gradient consequences}

Despite settling, such flows exhibit transport characteristics that are virtually indistinguishable from homogeneous visco-plastic flows. Consider, for example, the very coarse thickened mine water suspension shown in Figure 8 (Duckworth et al., 1986b).

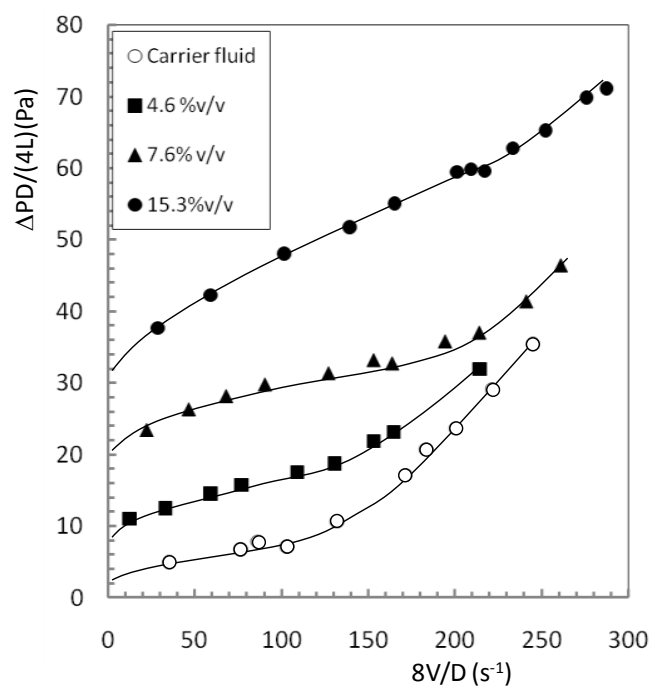

Figure 8 Pseudo-shear diagram for mine waste thickened tailings. Concentrations shown are for coarse particles added to the visco-plastic carrier fluid. Solid lines are laminar and turbulent curves based on pseudo-rheology

Apparent homogeneous fluid behaviour is displayed by the solid lines, based on the pseudo-rheology obtained from the laminar data in the pseudo-shear diagram. The curves shown are for Bingham model fluids, using Hank's method to predict the turbulent data (Darby and Melson, 1982). As shown, evidence of 
homogeneous flow is convincing, but - since the mine waste contained particles that are too coarse to form a non-Newtonian fluid - fallacious.

Because of such convincing behaviour, it is tempting to design the pipelines using this pseudo-rheology and conventional homogeneous flow formulae. If the flow is laminar, then such an approach will predict transport pressure gradients that are inversely proportional to pipe diameter. If, on the other hand, it is recognised that the flow will be stratified, a scaling that is essentially independent of pipe diameter, for industrially sized pipes, results (Newitt et al., 1955; Pullum, 2003; Pullum et al., 2004). Adopting the homogeneous model can result in the gross underestimation of the required pumping capabilities for industrially sized pipe.

\subsection{Inclined flows}

The effect of pipe incline can be ignored in homogeneous flows as the frictional head loss is independent of the pipe angle. All that is required to account for the effect of terrain on the total pressure gradient is the overall net head difference. For stratified flows, however, this is not the case and bed depth, the main contributor to frictional loss, is a function of pipe inclination, as seen in Figure 9.

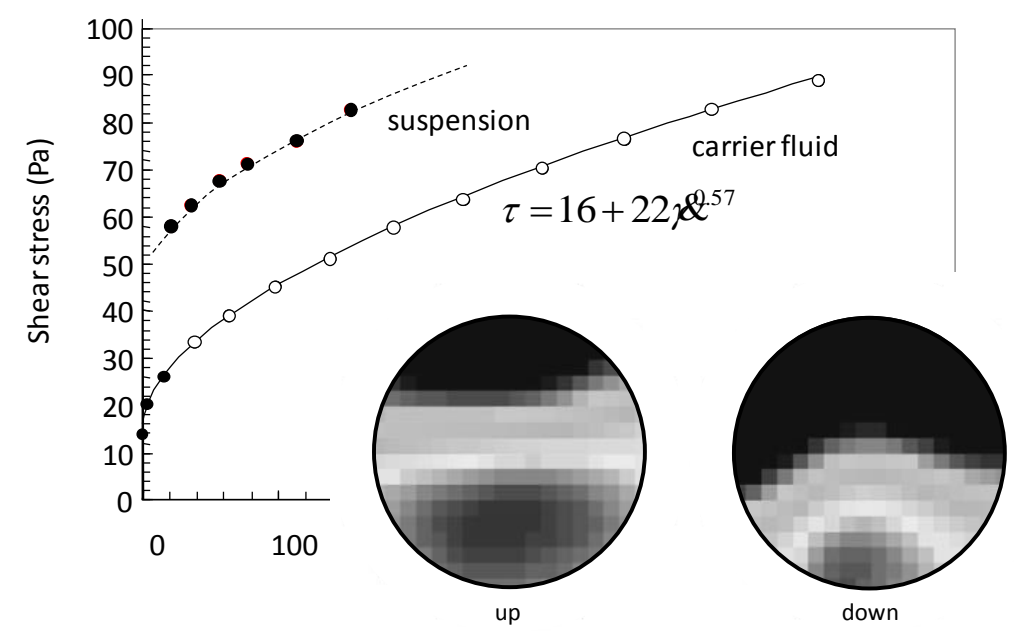

Figure 9 ERT concentration maps for coarse thickened suspension, showing the difference in the bed depth for positive and negatively inclined pipes (carrier fluid shown as dark grey in these images). The carrier fluid and suspensions rheograms are shown behind

Unsurprisingly, the bed depth is greater on flows going uphill, than on those going down, and consequently requires a higher transport gradient. While this variation in transport requirements is best determined by a suitable stratified model, a simple non-dimensional scaling model suitable for moderately inclined pipes, and which was given at an earlier conference in the series (Pullum et al., 2006), is repeated here. 


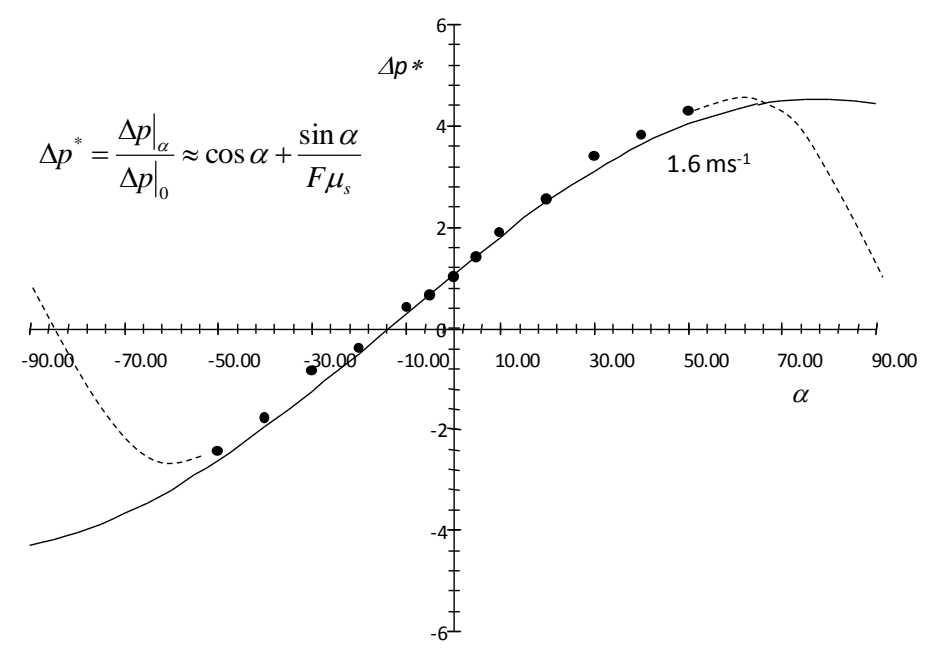

Figure 10 Variation of dimensionless pressure gradient $\not p *$ with pipe angle $\alpha_{0}$ Here the subscript refer to the pipe angle, $\mu_{s}$ is the Coulombic friction coefficient, associated with the bed and $F$ is model determined constant and varies from 1-2

This scaling factor is also applicable for non-Newtonian launders and sheet flows (Fuentes, 2007).

At higher angles, say $\alpha>+/-60^{\circ}$, the gradient decreases as the solids move into the main body of the flow, as indicated by the dashed lines in Figure 10.

\subsection{Vertical flow}

In vertical flow, the gravitational vector is axial and so the particles do not settle toward the pipe wall. Rather, they are lifted away from the wall, through the action of shear, to form a higher concentration of solids in the centre of the pipe, as shown in Figure 11.

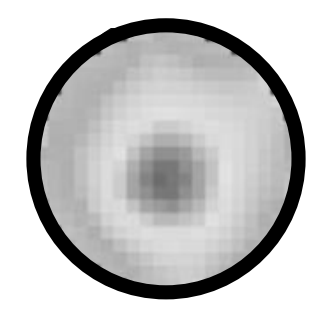

Figure 11 ERT concentration map showing particle migration into the central region of the pipe. (N.B. For this map the darker shades correspond to higher solids' concentrations)

The increase in frictional pressure drop, experienced in the horizontal and inclined lines, due to the bed, no longer exists and the frictional gradient can be calculated from the underlying carrier fluid's rheology and mixture density alone. Slip conditions can even occur in vertically downward flows.

\subsection{Bend flows}

The essentially axi-symetric concentration profile (Figure 11) is obtained when care is taken to ensure that the flow is introduced axially into the vertical pipe. For flows that travel around bends, the case is somewhat different. Consider a horizontal laminar, stratified flow, typical of thickened tailings, changing into a vertical flow through a bend. Secondary flows, induced in the bend, will act upon the bed, and, depending upon the strength of these secondary flows, will produce one of the three possible flow regimes depicted in Figure 12. 


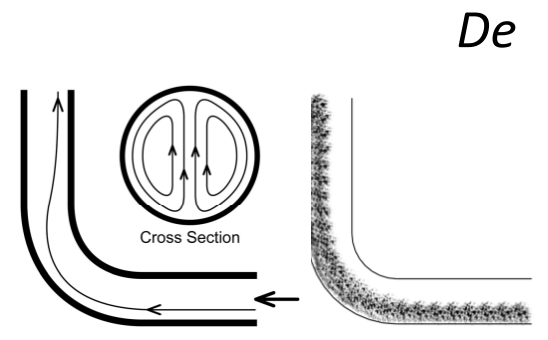

(a)

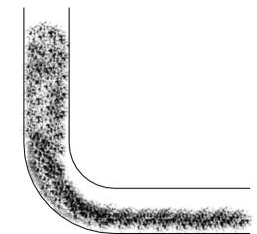

(b)

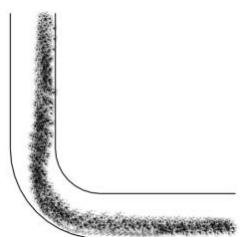

(c)

Figure 12 Flow regimes for stratified flow in a pipe bend. The three different regimes, (a), (b) and (c) are shown as a function of increasing Dean number, $D e$, where the Dean number is the product of the pipe Reynolds number and a ratio of the pipe radius and bend radius

- At low Dean numbers, regime (a), the secondary flow in the bend, is insufficient to modify the bed of solids substantially and the sliding bed of solids continues around the outer pipe wall.

- At moderate Dean numbers, regime (b), the secondary flows wrap the bed of solids around the inside of the pipe, to produce an axi-symmetric concentration map, where the central region is depleted of solids.

- At higher Dean numbers, regime (c), the secondary flow is sufficiently strong to transport the solids from the outer wall to the inner wall, within the transit of the bend.

Demonstration of these phenomena is shown in Figure 13 for a kaolin-based suspension.

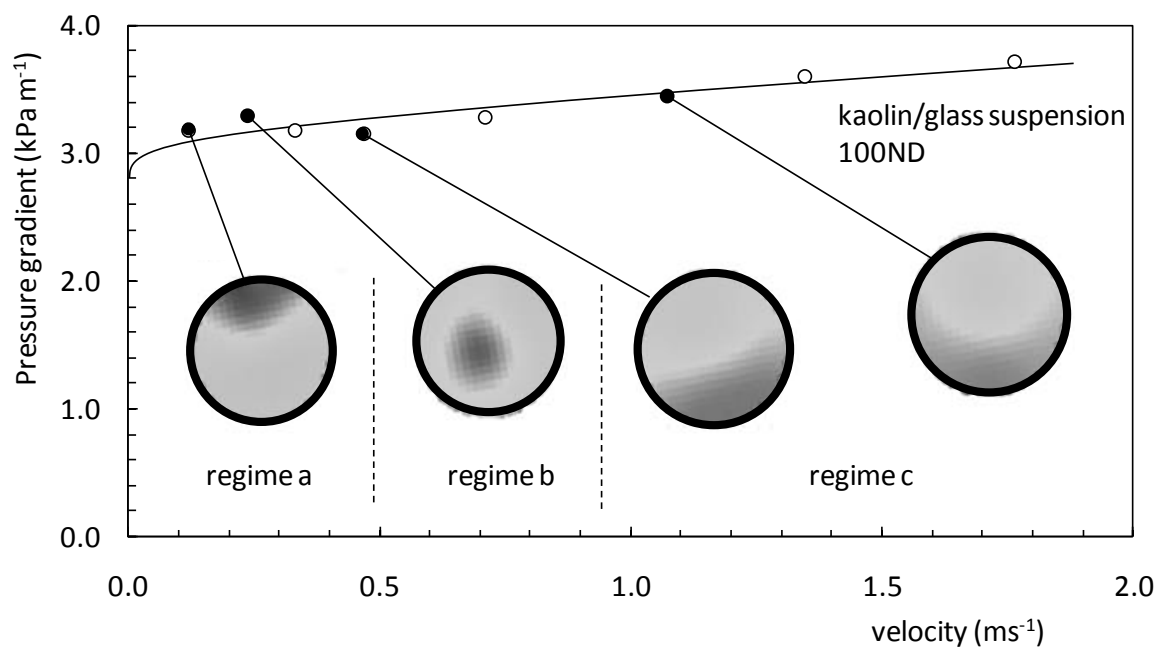

Figure 13 ERT maps, taken downstream of a vertical 1.5D bend, at various velocities showing the change in regime (N.B. for these maps the darker shades correspond to lower solids' concentrations)

Notice how the concentrations of the bed of solids, at velocities (Dean number) corresponding to regime (c), are more diffuse downstream of the bend, suggesting some form of reordering. Although not observed in vertical lines up to $100 \mathrm{D}$ long, it is expected that the flow regimes will eventually revert to ones similar to that shown in Figure 11, through the action of shear. It is also worth noting that suspensions that are statically stable, remain stable when the flow ceases, for regime (b), but produce slowly moving density currents back down the vertical pipe, for regimes (a) or (c) (Graham et al., 2010).

\subsection{Sheet flows and the TSF}

The discussions on sheared settling, given above for pipeline flows, apply equally to channel and sheet flows that are typically found in the tailings storage facility (TSF). Thickened tailings will be subject to 
stratification, due to the action of shear, and so beaching will be experienced, although nowhere near as dramatically as for conventional Newtonian systems. A typical coarse solids concentration profile measured in thickened tailings channel flow is shown in Figure 14 (Spelay, 2007).

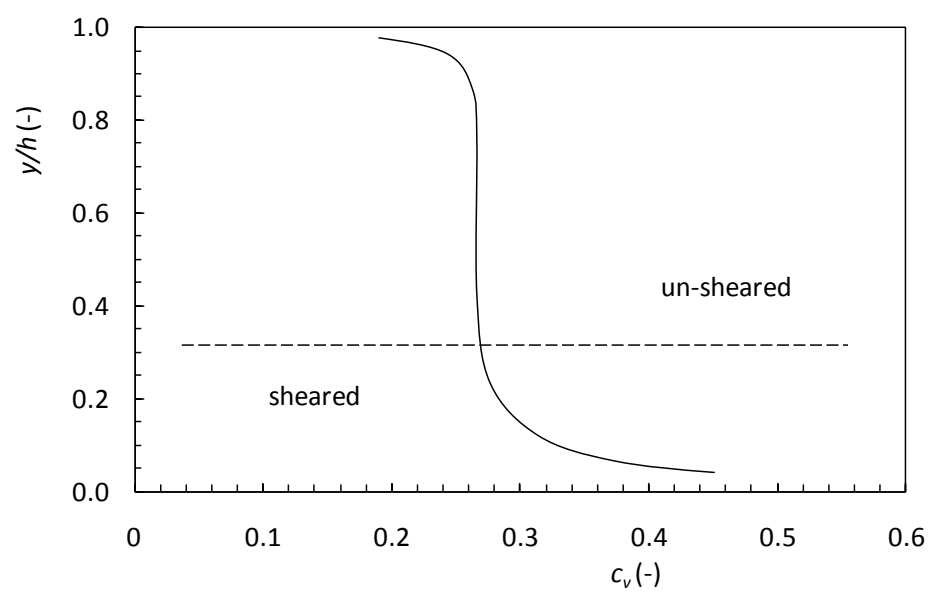

Figure 14 Typical concentration profile for channel flow of a thickened tailings containing a nominal $30 \%$ suspension of sand particles. Sand $d_{50}=188 \mathrm{~mm}$, channel slope $4.5^{\circ}$ (after Spelay, 2007)

The thickened suspensions, used in this study, were described as Bingham plastics and were statically stable, i.e. the coarse sand solids did not settle under stationary conditions. Flow was laminar and the channel semicircular in cross section; a reasonable approximation to channels found in TSFs (Chryss et al., 2006). The solids concentration is seen to be sensibly constant across the unsheared region of the flow, but settles in the sheared region through the same mechanisms as those described previously.

\section{Axial changes}

Most pastes and thickened tailings are thixotropic and, if they contain flocculants, will also suffer from permanent shear degradation (Barnes, 2001; Boger, 2009; Nguyen and Boger, 1985). As a consequence, if the material is not at its equilibrium condition, when it enters the tailings line, its structure will be further broken down by the shear in the pipe (Escudier and Presti, 1996; Pereira and Pinho, 2002) and if the material is homogeneous, the transport pressure gradient will reduce, as the material moves downstream.

If the flow is stratified, however, the picture is more complex. The sliding bed in a stratified flow, such as those described above, is moved by overcoming the bed's frictional resistance at the pipe wall and, by the combined effects of the pressure gradient across the bed and the drag of the fluid flowing over the bed. Since there is slip between the bed of solids and the flow over the bed, continuity demands a greater in situ concentration, or bed depth, for a given delivered concentration and slip velocity. The thixotropic nature of the fluid will mean that the fluid flowing over the bed will become less viscous, and hence, the drag upon the bed will be reduced. The in situ bed depth will therefore increase along the line, to compensate and will maintain the required force balance. Since, for industrially sized pipes, the pressure gradient is controlled by the bed depth, it is conceivable that the pressure gradient will increase, rather than decrease, along the line, due to the effects of thixotropy. This possible scenario is currently under investigation. 

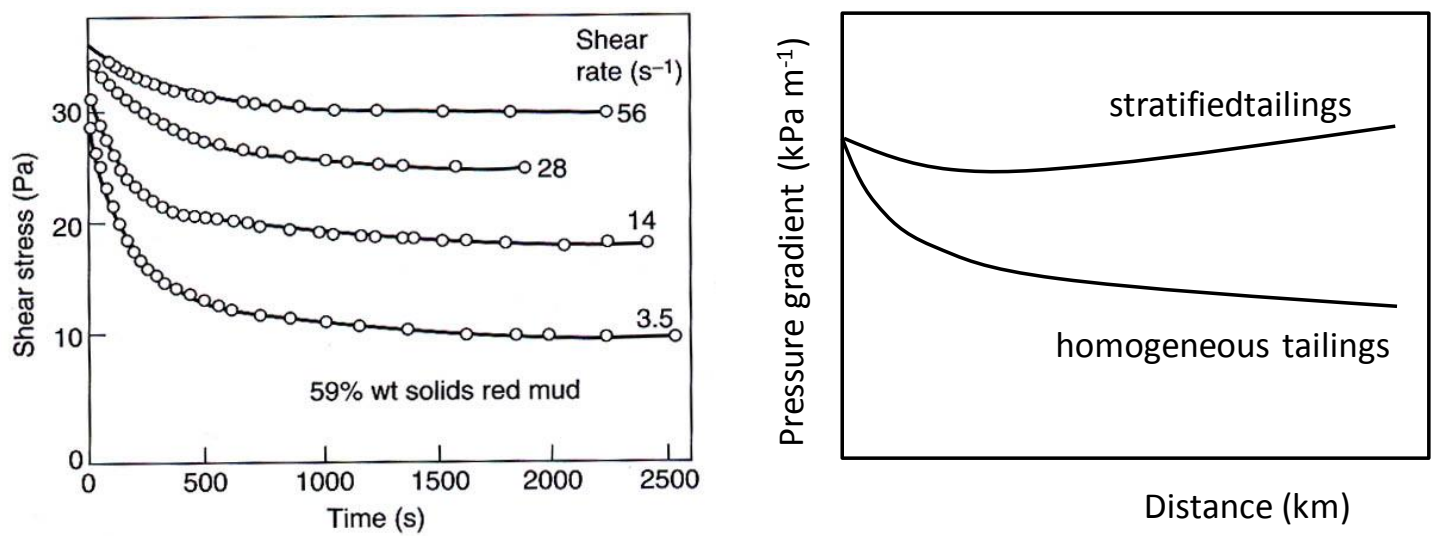

Figure 15 Typical thixotropic behaviour of thickened tailings slurry, and the possible pressure gradient profile for thixotropic tailings that may be considered to be either homogeneous or stratified

\section{$5 \quad$ Instrumentation and measurements of paste flows}

Instrumenting high concentration probes, such as pastes and thickened tailings, is very difficult. The flows are non-Newtonian, as we have seen, generally not homogeneous, and opaque. These conditions present a challenge, but recent developments in instrumentation techniques have enabled insights to be made.

\subsection{Imaging}

Traditionally the major problem with trying to understand these high concentration, solid fluid flows, has been the inability to build probes to investigate the internals of the flow that are, (i) robust enough to survive the savage environment, and (ii) small enough to provide information at sufficient resolution to avoid measuring "flow around the probe". The combination of advances in computational power, both embedded and local, and physics, has enabled a variety of relatively cheap, and robust, instrumentation to be developed, that can probe these flows unobtrusively. A very brief description of some of these enabling techniques, suitable for paste analysis, is given here, and the reader is referred to the literature for a more comprehensive description and analysis.

Ultrasonic Doppler Velocity Profiling (UVDP): This device produces a velocity profile, spatially distributed along an ultrasound beam that penetrates the flow. The flows must be particle laden. The device produces successful velocity profiles of the particles if the slip velocity between the particles and fluid is small (Elghobashi, 1994), although, where particle slip is high, the results are less reliable. Measurements have been successfully made in high concentration, non-Newtonian suspensions, and this technique is being considered as a means of providing rapid in-line viscometry (Wiklund et al., 2007; Wiklund and Stading, 2007). 


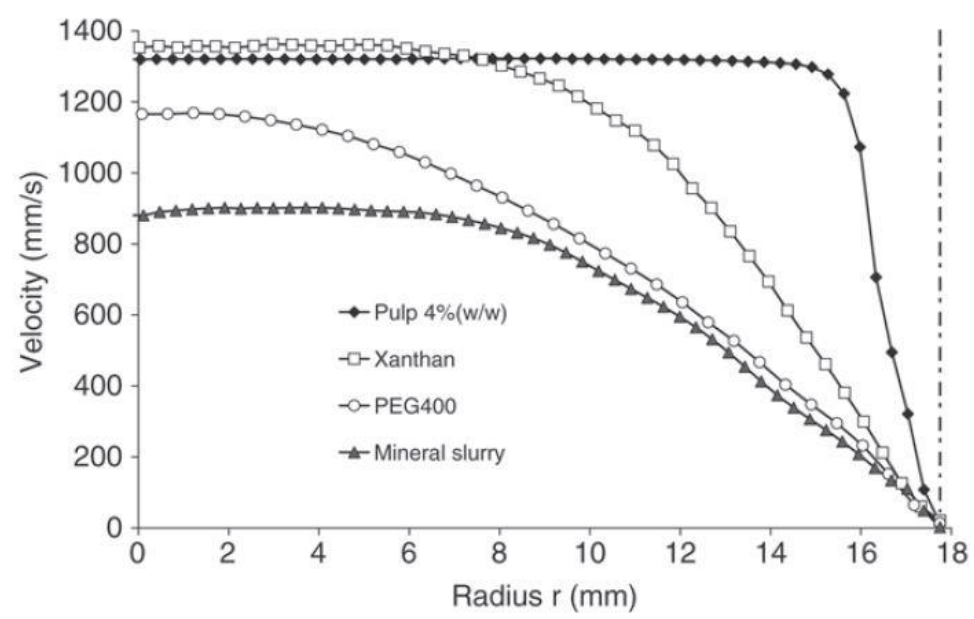

Figure 17 Velocity profile obtained in various opaque suspensions using UDVP (Wiklund and Stading, 2007)

Nuclear magnetic imaging (NMR or MRI): This high resolution, tomographic technique (Callaghan, 1991; Fukushima, 1999) is inherently sensitive to both concentration and velocity, enabling full 3D images of both variables to be measured, an example of which is given in Figure 6. Such tomographic imaging has been used to monitor particle migration in both laminar, Couette and Poiseuille suspension flows, as well as transitional and turbulent flows (Fukuda et al., 1985; Graham and Pullum, 2002; Jossic et al., 2002; Kose, 1991; Majors et al., 1989; Sinton and Chow, 1991). Despite its power, NMR suffers from extreme sensitivity to magnetic materials, e.g. fly ash suspensions contain too much magnetic iron from ball milling to be imaged and the equipment is delicate and requires cryogenic cooling to maintain the superconducting magnets that are used. Consequently, the equipment remains in the confines of well-equipped laboratories.

Electrical resistance tomography (ERT): Although not offering the spatial resolution of NMR, or radiometric tomographic techniques, ERT is a very simple and portable method that can be readily used to explore industrial type suspensions. The method relies on a difference in conductivity of the various phases to image them and provides rapid real time estimates of the 2D, transverse solids' conductivity, which is related to the solids' concentration through the Maxwell equation (e.g. http://www.itoms.com). Better resolved conductivity maps are achieved, if more complex reconstruction algorithms are used, example in the complex conjugate method (Wang, 2002). Examples of typical ERT tomograms for flows of pastes and thickened tailings are given in Figure 3 and Figure 9.

\subsection{Rheological parameters}

The forgoing analysis of the various flows suggests that successful modelling of these flows requires the rheological properties of the underlying carrier fluid, not the pseudo-rheology of the entire suspension. Vane tests are extensively used to measure the unsheared, or static yield stress, of pastes and high concentration suspensions, although such techniques are not guaranteed to give the correct values for all suspensions (Fourie and Dunn, 2007; Paterson, 2002). The presence of the coarse particles prevents this technique from measuring the underlying carrier fluid yield stress, which is closer to the actual value required for pipeline design (the sheared yield stress) as shown in Figure 16. 


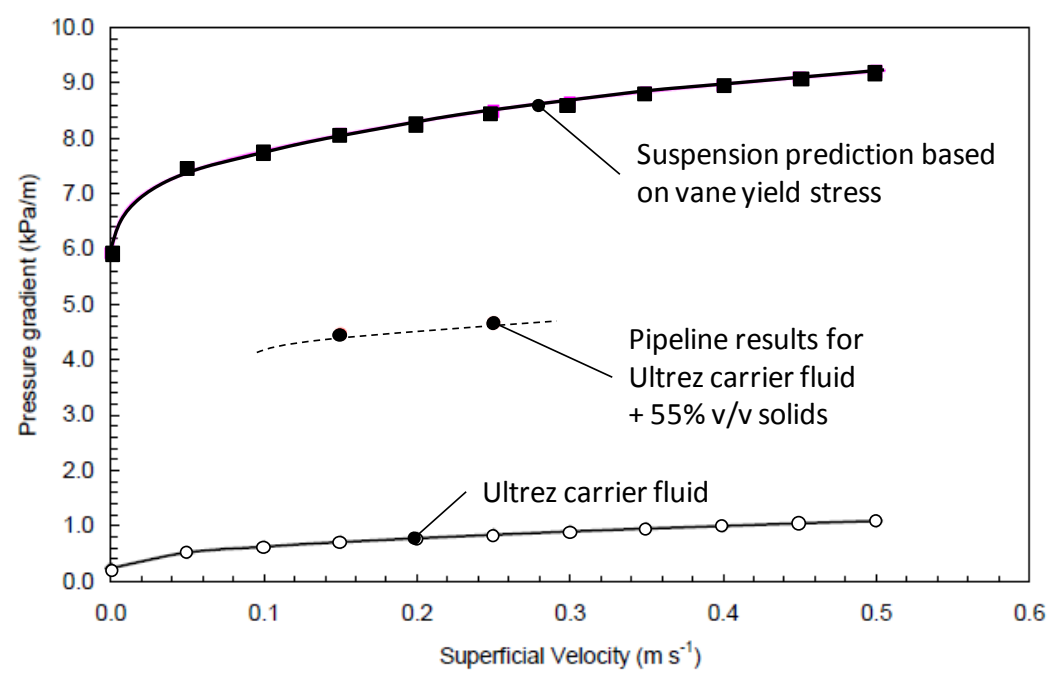

\section{Figure 16 Comparison between actual suspension behaviour and that predicted by vane measurement tests}

In this experiment, a suspension was made from a known carrier and a 55\% v/v suspension of inactive coarse particles. A vane test was conducted on the suspension in the normal manner and the yield stress noted. This yield stress was then used to predict the suspension's pipeline behaviour, based on this pseudo-rheology. The suspension was also pumped through a $100 \mathrm{~mm}$ test loop and the pressure drops measured. The values obtained were comparable with those predicted using a stratified model and the underlying carrier fluid rheology, whereas the pseudo-rheology based on the vane yield test overestimated the pipeline behaviour by $100 \%$. This demonstrates, either the inapplicability of this sort of test for pastes, where the coarse solids are conveyed in a stratified layer, or, the irrelevance of an indicated static yield stress in designing pipeline flow.

The question is raised as to how this underlying carrier fluid behaviour can be extracted from the suspensions? It was noted earlier, that for industrially sized pipes the pressure gradient is essentially insensitive to pipe diameter. This is because, at moderate to large pipe sizes, the pressure gradient is dominated by the bed. For small pipes, however, the opposite is true and the pressure gradient is dominated by the viscous nature of the carrier fluid. It can be shown by Pullum et al. (2010b) that the carrier fluid's rheology can be obtained by rudimentary removal of the coarse particles from the suspension and passing the scalped slurry through a small pipe viscometer. These properties are then used, in conjunction with a suitable stratified model, to predict the behaviour of the suspension in pipe sizes of interest.

\section{Conclusions}

Evidence has been presented which demonstrates that high concentration suspension flows, while appearing to behave as homogeneous non-Newtonian fluids are, in fact, generally stratified. Under static conditions the particles in the pastes and thickened tailings, that are too coarse to contribute to the rheology of the material, are held in suspension by the structured nature of the fluid, i.e. the yield stress. Once these materials are sheared, however, the fluid surrounding the particles dramatically drops in viscosity and the particles settle. The highly viscous nature of these flows means that the flows are laminar and, as such, forces countering this settling are small, if they exist at all. For horizontal and moderately inclined flows, this results in a stratified flow that imposes a large penalty on the frictional pressure gradient. Vertical flows do not suffer from this as the viscous nature of the flows aids in moving the coarser particles away from the wall.

Similar behaviour is observed in channel and sheet flows, implying that the deposition of these flows will also incur beaching effects that are similar, although nowhere near as extreme as conventional low concentration Newtonian systems. 
Although generally benign in behaviour, these suspensions have been shown to be quite complex, and the author hopes that design engineers will reconsider any design, based on simple homogeneous flow and the scale up of pseudo-rheological measurements, before implementing them.

\section{Acknowledgements}

The author would like to thank the CSIRO, Rio Tinto, the Weir Warman Group, De Beers and BHP Billiton for sponsoring much of the work reported here through the AMIRA P599 projects, and with especial thanks to my colleague Dr Lachlan Graham, at CSIRO, who was responsible for much of the imaging.

\section{References}

Barnes, H.A. (2001) Thixotropy - a review, Journal of Non-Newtonian Fluid Mechanics, Vol. 70, pp. 1-33.

Boger, D.V. (2009) Rheology and the resource industries, Chemical Engineering Science, Vol. 64, pp. 4525-4536.

Brown, N.P. (1988) Three scale-up techniques for stabilized coal-water slurries, Presented at the 11th International Conference on Hydrotransport, Stratford-upon-Avon, England, pp. 267-284.

Callaghan, P.T. (1991) Principles of Nuclear Magnetic Resonance Microscopy, Oxford Science Publications.

Chapman, B.K. and Leighton, D.T. (1991) Dynamic viscous re-suspension, International Journal Multiphase Flow, Vol. 17, pp. 469-483.

Charru, F. and Mouilleron-Arnould, H. (2002) Instability of a bed of particles sheared by a viscous flow, The Journal of Fluid Mechanics, Vol. 452, pp. 303-23.

Chryss, A.G., Fitton, T.G. and Bhatcaharya, S.N. (2006) Turbulent flow of non-Newtonian tailings in self formed channels on tailings stacks, in Proceedings Ninth International Seminar on Paste and Thickened Tailings (Paste06), R.J. Jewell, S. Lawson and P. Newman (eds), 3-7 April 2006, Limerick, Ireland, Australian Centre for Geomechanics, Perth, pp. 429-438.

Cooke, R. (2002) Laminar flow settling: the potential for unexpected problems, in Proceedings 15th International Conference on Hydrotransport, Banff, Canada, pp. 121-33.

Darby, R. and Melson, J.D. (1982) Direct Determinations of Optimum Economic Pipe Diameter for Non-Newtonian Fluids, Journal of Pipelines, pp. 11-21.

Duckworth, R.A., Addie, G.R. and Maffett, J.R. (1986a) Mine waste disposal by pipeline, Presented at the 11th International Conference on Slurry technology, Hilton Heads, South Carolina, USA.

Duckworth, R.A., Pullum, L. and Lockyear, C.F. (1982) The hydraulic transport of coarse coal at high concentrations, Journal of Pipelines, Vol. 4, pp. 251-65.

Duckworth, R.A., Pullum, L., Lockyear, C.F. and Addie, G.R. (1986b) The pipeline transport of coarse materials in a non-Newtonian carrier fluid, in Proceedings 10th International Conference on the Hydraulic Transport of Solids in Pipes, Innsbruck, Austria, pp. 69-88.

Elghobashi, S. (1994) On predicting particle-laden turbulent flows, Applied Scientific Research, Vol. 52, pp. 309-29.

Escudier, M.P. and Presti, F. (1996) Pipe flow of a thixotropic liquid, Journal of non-Newtonian Fluid Dynamics, Vol. 62, pp. 291-306.

Fourie, A.B. and Dunn, F. (2007) Limitations to the use of the modified slump test for yield stress determination, in Proceedings Tenth International Seminar on Paste and Thickened Tailings (Paste07), A.B. Fourie and R.J. Jewell (eds), 13-15 March 2007, Perth, Australia, Australian Centre for Geomechanics, Perth, pp. 216-228.

Fukuda, K., Inouye, A., Kawabe, Y. and Hirai, A. (1985) Transition from Laminar to Turbulent Flow of Water a Pipe Measured by a Pulsed NMR Method, Journal of the Physical Society of Japan, Vol. 54, pp. 4555-4560.

Fukushima, E. (1999) Nuclear magnetic resonance as a tool to study flow, Annual Review of Fluid Mechanics, Vol. 31, pp. $95-123$.

Graham, L.J.W. and Pullum, L. (2002) An investigation of complex hybrid suspension flows by magnetic resonance imaging, Canadian Journal of Chemical Engineering, Vol. 80, pp. 200-207.

Graham, L.J.W., Wu, J. and Pullum, L. (2010) Vertical conveying of coarse particles in pipelines with non-Newtonian carriers, in Proceedings 18th International Conference on Hydrotransport, Rio de Janeiro, Brazil, pp. 67-80.

Hou, H.C. (1986) Investigation of optimal grain distribution for transport with high concentration, in Proceedings 10th International Conference on Hydrotransport, Innsbruck, Austria, pp. 177-184.

Jossic, L., Briguet, A. and Magnin, A. (2002). Segregation under flow of objects suspended in a yield stress fluid and NMR imaging visualisation, Chemical Engineering Science, pp. 409-418.

Kose, K. (1991) Instantaneous flow-distribution measurements of the equilibrium turbulent region in a circular pipe using ultrafast NMR imaging, Physical review, Vol. A44, pp. 2495-2504.

Krishnan, G.P. and Leighton Jr., D.T. (1995) Dynamic viscous re-suspension of bi-disperse suspensions, Effective diffusivity, Heat and Mass Transfer, Vol. 21, pp. 526-526. 
Lawler, H.L., Cowper, N.T., Pertuit, P. and Tennant, J.D. (1978) Application of stabilised slurry concepts of pipeline transportation of large particle coal, Presented at the 3rd International Technical Conference on Slurry Transportation, Las Vegas, Nevada.

Leighton, D. and Acrivos, A. (1986) Viscous re-suspension, Chemical Engineering Science, Vol. 41, pp. $1377-1384$.

Lockyear, C.F., Pullum, L., Duckworth, R.A., Littlejohn, M.H. and Lenard, J.A. (1984) The rheology of fine coal suspensions, Presented at the Transportation Conference, Perth, Australia.

Majors, P.D., Givler, R.C. and Fukushima, E. (1989) Velocity and Concentration Measurements in Multiphase Flows by NMR, Journal of Magnetic Resonance, Vol. 85, pp. 235-243.

Newitt, R.D.M., Richardson, J.F., Abbott, M. and Turtle, R.B. (1955) Hydraulic conveying of solids in horizontal pipes, Transactions of the American Institute of Chemical Engineers, Vol. 33, pp. 93-113.

Nguyen, Q.D. and Boger, D.V. (1985) Thixotropic behaviour of concentrated bauxite residue suspensions, Rheolgica Acta, Vol. 24, pp. 427-37.

Paterson, A.J.C. (2002) Is slump a valid measure of the rheological properties of high concentration paste slurries?, in Proceedings 15th International Conference on Hydrotransport, Banff, Canada, pp. 361-374.

Pereira, A.S. and Pinho, F.T. (2002) Turbulent pipe flow of thixotropic fluids. International Journal Heat and Fluid Flow, Vol. 23, pp. 36-51.

Pullum, L. (2003) Pipeline performance, in Proceedings Paste 2003, Melbourne, Australia, pp. Sect.8:1-13.

Pullum, L. and Graham, L.J.W. (1999) A new high-concentration pipeline test loop, Presented at 14th International Conference on Hydrotransport, Maastricht, Netherlands, pp. 505-514

Pullum, L., Graham, L.J.W., Rudman, M., Aldham, B. and Hamilton, R. (2006) The ups and downs of paste transport, in Proceedings Ninth International Seminar on Paste and Thickened Tailings (Paste06), R.J. Jewell, S. Lawson and P. Newman (eds), 3-7 April 2006, Limerick, Ireland, Australian Centre for Geomechanics, Perth, pp. 395-402.

Pullum, L., Graham, L.J.W. and Slatter, P. (2004) A non-Newtonian two-layer model and its application to high density transport, in Proceedings 16th International Conference on Hydrotransport, Santiago, Chile, pp. 579-593.

Pullum, L., Graham, L.J.W. and Wu, J. (2010a) Bed establishment lengths under laminar flow, in Proceedings 18th International Conference on Hydrotransport, Rio de Janeiro, Brazil, pp. 261-274.

Pullum, L. and Littlejohn, M.H. (1986) Coarse coal/water slurry transport, Presented at the Australian Society of Rheology: Concentrated Slurry Technology conference, Melbourne.

Pullum, L., Slatter, P., Graham, L. and Chryss, A. (2010b) Are tube viscometer data valid for suspension flows?, KoreaAustralia Rheology Journal, Vol. 22, pp. 225-230.

Schaflinger, U., Acrivos, A. and Stibi, H. (1995) An experimental study of viscous re-suspension in a pressure-driven plane channel flow. International Journal of Multiphase Flows 21, pp. 693-704.

Schaflinger, U., Acrivos, A. and Zhang, K. (1990) Viscous re-suspension of a sediment within a laminar and stratified flow, International Journal Multiphase Flow, Vol. 16, pp. 567-578.

Sinton, S.W. and Chow, A.W. (1991) NMR flow imaging of fluids and solid suspensions in Poiseuille flow, Journal of Rheology, Vol. J5, pp. 735-55.

Spelay, R.B. (2007) Solids transport in laminar, open channel flow of non-Newtonian slurries, Doctoral thesis, Department of Chemical Engineering University of Saskatchewan, Saskatoon, Canada.

Talmon, A. and Mastbergen, D. (2004) Solids transport by drilling fluids: Cobcentrated bentonite-sand-slurries, in Proceedings 12th Transport and Sedimentation of Solid Particles, Prague, Czech Republic, pp. 641-49.

Thomas, A.D. (1979) Settling of particles in a horizontally sheared Bingham plastic, Presented at the First National Conference on Rheology, RMIT, Melbourne, Australia.

Thomas, A.D., Pullum, L. and Wilson, K.C. (2004) Stabilised laminar slurry flow: review, trends and prognosis, in Proceedings 16th International Conference on Hydrotransport., Santiago, Chile, pp. 701-716.

Tripathi, A. and Acrivos, A. (1999) Viscous re-suspension in a bi-density suspension, International Journal of Multiphase Flows, Vol. 25, pp. 1-14.

Wang, M. (2002) Inverse solution of electrical impedance tomography based on conjugate gradient methods, Measurement Science and Technology, Vol. 13, pp. 101-117.

Wiklund, J., Shahram, I. and Stading, M. (2007) Methodology for in-line rheology by ultrasound Doppler velocity profiling and pressure difference techniques, Chemical Engineering Science, Vol. 62, pp. 4277-4293.

Wiklund, J. and Stading, M. (2007) Application of in-line ultrasound Doppler-based UVP-PD rheometry method to concentrated model and industrial suspensions, ISUD5: The 5th International Symposium on Ultrasonic Doppler Methods for Fluid Mechanics and Fluid Engineering, 19, pp. 171-179.

Wilson, K.C. and Horsley, R.R. (2004) Calculating fall velocities in non-Newtonian (and Newtonian) fluids: a new view, in Proceedings 16th International Conference on Hydrotransport, Santiago, Chile, pp. 37-46.

Zhang, K. and Acrivos, A. (1994) Viscous re-suspension in fully developed laminar pipe flows, International Journal Multiphase Flow, Vol. 20, pp. 579-91. 
